\title{
Anopheles gambiae eicosanoids modulate Plasmodium berghei survival from oocyst to salivary gland invasion
}

\author{
Susana Ramos, Ana Custódio, Henrique Silveira/ ${ }^{+}$ \\ Centro de Malária e Outras Doenças Tropicais, Unidade de Ensino e Investigação de Parasitologia Médica, \\ Instituto de Higiene e Medicina Tropical, Universidade Nova de Lisboa, Lisboa, Portugal
}

Eicosanoids affect the immunity of several pathogen/insect models, but their role on the Anopheles gambiae response to Plasmodium is still unknown. Plasmodium berghei-infected mosquitoes were injected with an eicosanoid biosynthesis inhibitor, indomethacin (IN), or a substrate, arachidonic acid (AA), at day 7 or day 12 post-infection (p.i.). Salivary gland invasion was evaluated by sporozoite counts at day 21 p.i. IN promoted infection upon sporozoite release from oocysts, but inhibited infection when sporozoites were still maturing within the oocysts, as observed by a reduction in the number of sporozoites reaching the salivary glands. AA treatment had the opposite effect. We show for the first time that An. gambiae can modulate parasite survival through eicosanoids by exerting an antagonistic or agonistic effect on the parasite, depending on its stage of development.

Key words: eicosanoid - Anopheles - Plasmodium

Anopheles mosquitoes are known to respond to Plasmodium infection by activating their immune system. To date, research has mainly focused on the response to midgut invasion by ookinetes, whereas other aspects of the response throughout the sporogonic cycle are scarce, even though only approximately $2 \%$ of the sporozoites released from the midgut effectively invade the salivary glands (Hillyer et al. 2007). A reduction in parasite number in the haemolymph can be the result of physical barriers (reducing the success of reaching the salivary glands), immunity (parasite clearance) or of mechanisms involving mosquito physiology.

The gene retinoid and fatty-acid binding glycoprotein codes for a precursor of apolipophorin I and II, which are the two major components of the insect lipid transporter. The expression of this gene is highly induced upon ookinete invasion and its knock-down leads to a reduction in oocyst number and to the total inhibition of egg development (Vlachou et al. 2005). This suggests that responses to pathogens may include the action of not only molecules previously described as immune related, but also physiological and general mechanisms operating in the mosquito.

Eicosanoid biosynthesis, in particular, has been implicated in the responses of several insects [reviewed by Stanley and Kim (2014)]. Eicosanoids are oxygenated metabolites of arachidonic acid (AA) and other polyun-

doi: 10.1590/0074-0276140098

Financial support: POCI/SAU-IMI/59489/2004, PTDC/SAUMII/102596/2008

SR was the recipient of FCT (SFRH/BD/12210/2003) and HS is a recipient of a PVE (Programa Ciência sem Fronteira FMT-HVD, Brazil)

+ Corresponding author: hsilveira@ihmt.unl.pt

Received 20 March 2014

Accepted 30 June 2014 saturated fatty acids that act mainly as signalling molecules, such as ovulatory pheromones and modulators of ion transport.

Membrane phospholipids are hydrolysed by phospholipase A2 (PLA2) into AA, which is subsequently metabolised into eicosanoids by three pathways: cyclooxygenases (COX) convert AA into prostaglandins (PGs), thromboxanes and prostacyclin, lipoxygenases (LOX) convert AA into leukotrienes, hydroxy fatty acids, lipoxins and hepoxillins and cytochrome $\mathrm{P} 450$ produces hydroxy fatty acids and epoxy derivatives. In insects, eicosanoid biosynthesis has been implicated in the response to bacteria, fungi and parasites, as treatment with chemical inhibitors of the enzymes involved in this process results in a compromised immune response. Dexamethasone (DEX), a PLA2 inhibitor, was shown to reduce the haemocyte microaggregation and nodulation response of Manduca sexta to the bacterium Serratia marcescens, effects that were reversed by AA treatment (Miller et al. 1994). During bacterial infections, eicosanoids also mediate phagocytosis, cell spreading, prophenoloxidase (PPO) activation and, in Drosophila, immunedeficiency pathway activation (Miller et al. 1994, Mandato et al. 1997, Yajima et al. 2003). In $M$. sexta infected with the fungus Metarhizium anisopliae or Beauveria bassiana, products of the LOX, but not the COX pathway were found to reduce the nodulation response (Dean et al. 2002, Lord et al. 2002).

With regard to parasite infections, DEX treatment of Drosophila larvae inhibits the encapsulation of Leptopilina boulardi eggs (Carton et al. 2002) and increased mortality by Heterorhabditis bacteriophora, which was associated with the inhibition of microclot formation (Hyrsl et al. 2010). In the model Rhodnius prolixus/ Trypanosoma rangeli, treatment with DEX, indomethacin (IN) (COX inhibitor) or nordihydroguaiaretic acid (a non-specific LOX inhibitor) completely abolishes the microaggregation response and greatly reduces PPO activation while increasing parasite numbers in the haemo- 
lymph. These effects are reversed by AA treatment, suggesting that eicosanoids modulate this response (Garcia et al. 2004a). It was also shown that in Anopheles albimanus, a primary vector for malaria vivax in Mexico, DEX inhibits the expression of mRNAs corresponding to specific antimicrobial peptides (AMPs), such as cecropin, gambicin and attacin (García-Gil-de-Muñoz et al. 2008).

Bearing this information in mind, we hypothesised that eicosanoid biosynthesis is altered during mosquito infection and plays a role in the mosquito response to sporozoites in the haemolymph. To test this hypothesis, Plasmodium berghei-infected mosquitoes were injected with either an eicosanoid biosynthesis inhibitor (IN, Fluka, Sigma) or a substrate (AA, Fluka, Sigma). Both compounds were used to prepare solutions of $0.1,1$ and 10 $\mathrm{mg} / \mathrm{mL}$ in Schneider medium (Sigma) with $6 \%$ ethanol. The toxicity of IN and AA to the mosquitoes was tested by injection of $69 \mathrm{nl}$ of each solution into Anopheles gambiae Yaoundé female mosquitoes using a Nanojet II (Drummond Scientific). For each group, 20 mosquitoes were injected intrathoraxically with $69 \mathrm{nl}$ of the respective solution and then compared with controls. Survival was determined by counting dead mosquitoes for seven days and the data were analysed using a Mantel-Cox Log-Rank test. There were no differences in mortality between the groups tested (Fig. 1).

To evaluate the influence of IN and AA on infection, three-five-day-old mosquitoes were allowed to feed on mice infected with $P$. berghei ANKA. At day 7 postinfection (p.i.), when the oocysts were still developing, the infected mosquitoes $(\sim 100$ +) were injected with Schneider medium plus $10 \%$ ethanol (control group), IN at $1 \mathrm{mg} / \mathrm{mL}$ (IN group) or AA at $1 \mathrm{mg} / \mathrm{mL}$ (AA group). Other $P$. berghei-infected mosquitoes were also injected with control solution, IN or AA at day 12 p.i., which corresponds to the time the majority of oocysts are developed and the sporozoites are starting to egress to the haemolymph. Three replicates were performed for each experiment. All mosquitoes were dissected at day 21 p.i. and salivary glands were placed in a glass homogeniser and crushed to release the sporozoites; the sample was

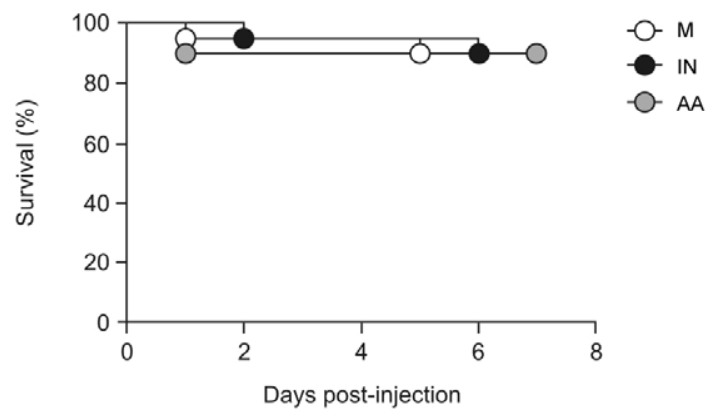

Fig. 1: mosquito survival. Mosquitoes were injected either with indomethacin $(I N) 1 \mathrm{mg} / \mathrm{mL})(\mathrm{n}=20)$ or arachidonic acid $(A A)(1 \mathrm{mg} / \mathrm{mL})$ $(\mathrm{n}=20)$ in Schneider medium with $6 \%$ ethanol. Control mosquitoes were injected with Schneider medium with $6 \%$ ethanol $(n=20)$. Dead mosquitoes were counted in each group for seven days. Survival was analysed using the Log-rank (Mantel-Cox) test. M: control. mixed with $400 \mu \mathrm{L}$ of phosphate buffered saline. The number of sporozoites in the suspension was counted using a haematocytometer. The data were evaluated for statistical significance by applying a ratio $t$ test using the GraphPad software (Prism).

Treatment of the infected mosquitoes with the inhibitor (IN) resulted in a decreased number of sporozoites recovered from the salivary glands when inhibition was performed at day 7 p.i., whereas an increase was observed when the inhibitor was used at day 12 p.i. (Fig. 2A, B). The opposite trend was observed when the eicosanoid biosynthesis substrate (AA) was injected (Fig. 2C, D). The injection of AA at day 12 p.i. led to a significant reduction in salivary gland sporozoite number to half of that found in the control mosquitoes (Fig. 2D), confirming that eicosanoids are necessary to clear sporozoites from the mosquito haemolymph. Additionally, at day 7 p.i., a 1.7-fold increase was observed in the parasite number in the salivary glands of the AA-injected mosquitoes when compared to the controls (Fig. 2C), corroborating that eicosanoids are at some point required for parasite development. This may reflect a role for mosquito-synthesised eicosanoids in Plasmodium biology and development.

An increase in parasite development in oocysts might reflect a parasitic metabolic need for lipids. Although the parasite has its own eicosanoid biosynthetic pathways, which are different from those present in mammals, it is not capable of the de novo synthesis of fatty acids, requiring AA from its host (Holz 1977). Furthermore, interactions of oocysts with AA might alter the lipid composition in the oocyst membrane, thereby influenc-
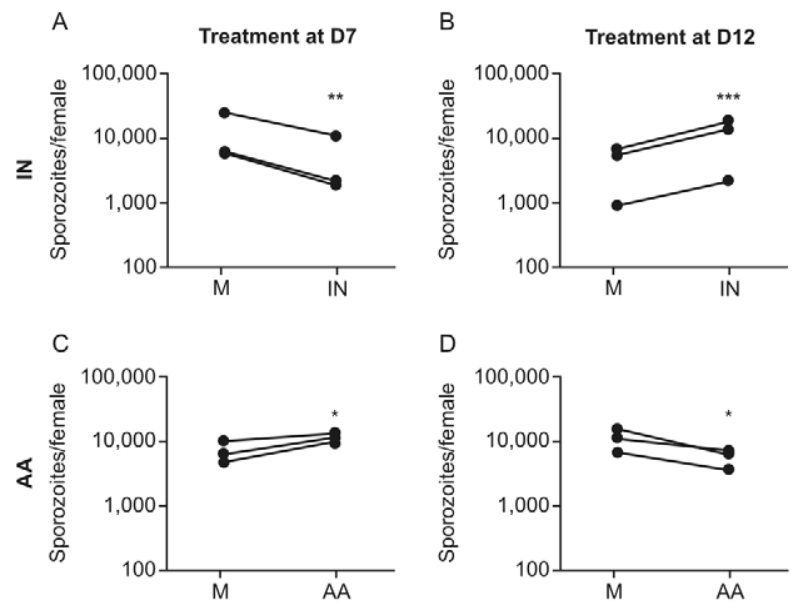

Fig. 2: the impact of eicosanoid biosynthesis on the outcome of Plasmodium berghei infection in Anopheles gambiae mosquitoes. P. berghei infected mosquitoes were injected at day (D) 7 (left panel) or D12 (right panel) with indomethacin (IN) $(1 \mathrm{mg} / \mathrm{mL}$ ) (upper panel), arachidonic acid (AA) $(1 \mathrm{mg} / \mathrm{mL})$ (lower panel) or Schneider medium with $6 \%$ ethanol (M). P. berghei sporozoites were collected and counted at D21 post-infection and are represented as average number of sporozoites per female in each group. Each line represents an independent experiment. Data is derived from three independent experiments with the same trend. Differences were evaluated using a ratio $t$ test, with the GraphPad software (Prism). *: $\mathrm{p}<0.05 ; * *: \mathrm{p}<0.001 ; * * * \mathrm{p}<0.0001$. 
ing recognition by the immune system. However, there is also some evidence that points to a role for eicosanoids in controlling phase changes and differentiation in protozoa (Noverr et al. 2003). Nevertheless, our data do not allow confirmation of any of these hypotheses.

In vertebrates, eicosanoids mediate inflammation and immune responses, having roles in platelet aggregation, intensity and duration of pain and fever and blood pressure regulation. In insects, eicosanoid biosynthesis has also been linked to immune responses. Indeed, accumulating data suggest that eicosanoids have an important role in mediating responses to bacterial, fungal and parasite infections in six orders of insects [reviewed by Stanley and Kim (2014)].

The fact that AA injection boosted the mosquito haemolymph response to sporozoites suggests that eicosanoid biosynthesis in infected mosquitoes is not operating at a maximum level. One possible explanation would be an ability of the parasite to immunosuppress its mosquito host. The ability of the parasite to evade and/ or suppress the host's immune response has been extensively studied in vertebrate hosts. In the blood stages of the $P$. falciparum life cycle, haemozoin was shown to be responsible for the inhibition of human PGE2 gene expression, the reduced levels of which led to a subsequent increase in tumour necrosis factor alpha levels, accounting for anaemia (Keller et al. 2004, 2006). In its mosquito vector, Plasmodium was also shown to possess immune evasion and suppression behaviours. In late oocyst stages, parasites are believed to camouflage themselves by incorporating mosquito-derived proteins into their surface and, in early stages, become wrapped in the mosquito's plasma membrane when traversing the midgut epithelium (Vlachou et al. 2004).

Some mosquito proteins, such as CTL4 and CTLMA2, were found to be protective for the parasite, possibly assisting in immune evasion. Furthermore, midgut stages of Plasmodium gallinaceum reduce the ability of Aedes aegypti to encapsulate Sephadex beads (Boete et al. 2004) and P. falciparum infection was found to repress the expression of nitric oxide synthase, a gene involved in local epithelial responses (Tahar et al. 2002).

An intriguing property of host immunosuppression by pathogens has been observed in two models of infection in insects, including both bacterial and parasite infections that compromise the respective host's immunity by interfering with eicosanoid biosynthesis. The bacterium Xenorhabdus nematophila is able to inhibit Spodoptera exigua PLA2 activity, as the injection of AA into $S$. exigua larvae reduces host mortality by $50 \%$; in contrast, treatment with inhibitors potentiates bacterial mortality (Park \& Kim 2000, 2003). This inhibition accounts for a specific time frame of cellular immunity inhibition (Eom et al. 2014) and AMP production suppression (Hwang et al. 2013). Moreover, in a protozoan infection, $T$. rangeli in $R$. prolixus, AA treatment increases the microaggregation reaction and reduces parasite numbers in the insect's haemolymph. T. rangeli was proposed to inhibit the release of AA in its host, though it is not known whether the parasite has a direct action on PLA2 (Garcia et al. 2004b). In A. gambiae, eicosa- noids have a dual role on the outcome of Plasmodium infection, promoting parasite development in the oocyst stage or controlling infection when parasites are released to the haemolymph.

The maintenance and care of experimental animals was carried out in accordance with the European Directive 86/609/European Economic Community and Portuguese law (129/92) for biomedical research involving animals. Full details of this study were approved by the Veterinary General Directorate, Portugal (ordinance $8-1005 / 92,23 / 2010)$.

\section{ACKNOWLEDGEMENTS}

To Catarina Alves, for mosquito rearing.

\section{REFERENCES}

Boete C, Paul RE, Koella JC 2004. Direct and indirect immunosuppression by a malaria parasite in its mosquito vector. Proc Biol Sci 271: 1611-1615.

Carton Y, Frey F, Stanley DW, Vass E, Nappi AJ 2002. Dexamethasone inhibition of the cellular immune response of Drosophila melanogaster against a parasitoid. J Parasitol 88: 405-407.

Dean P, Gadsden JC, Richards EH, Edward JP, Charnley Ak, Reynolds SE 2002. Modulation by eicosanoid biosynthesis inhibitors of immune responses by the insect Manduca sexta to the pathogenic fungus Metarhizium anisopliae. J Invertebr Pathol 79: 93-101.

Eom S, Park Y, Kim Y 2014. Sequential immunosuppressive activities of bacterial secondary metabolites from the entomopahogenic bacterium Xenorhabdus nematophila. J Microbiol 52: 161-168.

Garcia ES, Machado EM, Azambuja P 2004a. Effects of eicosanoid biosynthesis inhibitors on the prophenoloxidase-activating system and microaggregation reactions in the hemolymph of Rhodnius prolixus infected with Trypanosoma rangeli. J Insect Physiol 50: 157-165.

Garcia ES, Machado EM, Azambuja P 2004b. Inhibition of hemocyte microaggregation reactions in Rhodnius prolixus larvae orally infected with Trypanosoma rangeli. Exp Parasitol 107: 31-38.

García-Gil-de-Muñoz FL, Martínez-Barnetche J, Lanz-Mendoza H, Rodríguez MH, Hernández-Hernández FC 2008. Prostaglandin E2 modulates the expression of antimicrobial peptides in the fat body and midgut of Anopheles albimanus. Arch Insect Biochem Physiol 68: 14-25.

Hillyer JF, Barreau C, Vernick KD 2007. Efficiency of salivary gland invasion by malaria sporozoites is controlled by rapid sporozoite destruction in the mosquito haemocoel. Int J Parasitol 37: 673-681.

Holz GG, Beach DH, Sherman IW 1977. Octadecenoic fatty acids and their association with hemolysis in malaria. J Protozool 24: 566-574.

Hwang J, Park Y, Kim Y, Hwang J, Lee D 2013. An entomopathogenic bacterium, Xenorhabdus nematophila, suppresses expression of antimicrobial peptides controlled by Toll and IMD pathways by blocking eicosanoid biosynthesis. Arch Insect Biochem Physiol 83: 151-169.

Hyrsl P, Dobes P, Wang Z, Hauling T, Wilhelmsson C, Theopold U 2010. Clotting factors and eicosanoids protect against nematode infections. J Innate Immun 3: 65-70.

Keller CC, Davenport GC, Dickman KR, Hittner JB, Kaplan SS, Weinberg JB, Kremsner PG, Perkins DJ 2006. Suppression of prostaglandin E2 by malaria parasite products and antipyretics promotes overproduction of tumor necrosis factor-alpha: association with the pathogenesis of childhood malarial anemia. J Infect Dis 193: 1384-1393. 
Keller CC, Hittner JB, Nti BK, Weinberg JB, Kremsner PG, Perkins DJ 2004. Reduced peripheral PGE2 biosynthesis in Plasmodium falciparum malaria occurs through hemozoin-induced suppression of blood mononuclear cell cyclooxygenase-2 gene expression via an interleukin-10-independent mechanism. Mol Med 10: 45-54.

Lord JC, Anderson S, Stanley DW 2002. Eicosanoids mediate Manduca sexta cellular response to the fungal pathogen Beauveria bassiana: a role for the lipoxygenase pathway. Arch Insect Biochem Physiol 51: 46-54.

Mandato CA, Diehl-Jones WL, Moore SJ, Downer RG 1997. The effects of eicosanoid biosynthesis inhibitors on prophenoloxidase activation, phagocytosis and cell spreading in galleria mellonella. J Insect Physiol 43: 1-8.

Miller JS, Nguyen T, Stanley-Samuelson DW 1994. Eicosanoids mediate insect nodulation responses to bacterial infections. Proc Natl Acad Sci USA 91: 12418-12422.

Noverr MC, Erb-Downward JR, Huffnagle GB 2003. Production of eicosanoids and other oxylipins by pathogenic eukaryotic microbes. Clin Microbiol Rev 16: 517-533.

Park Y, Kim Y 2000. Eicosanoids rescue Spodoptera exigua infected with Xenorhabdus nematophilus, the symbiotic bacteria to the entomopathogenic nematode Steinernema carpocapsae. J Insect Physiol 46: 1469-1476.

Park Y, Kim Y 2003. Xenorhabdus nematophilus inhibits p-bromophenacyl bromide (BPB)-sensitive PLA2 of Spodoptera exigua. Arch Insect Biochem Physiol 54: 134-142.

Stanley D, Kim Y 2014. Eicosanoid signaling in insects: from discovery to plant protection. CRC Crit Rev Plant Sci 33: 20-63.

Tahar R, Boudin C, Thiery I, Bourgouin C 2002. Immune response of Anopheles gambiae to the early sporogonic stages of the human malaria parasite Plasmodium falciparum. EMBO J 21: 6673-6680.

Vlachou D, Schlegelmilch T, Christophides GK, Kafatos FC 2005. Functional genomic analysis of midgut epithelial responses in Anopheles during Plasmodium invasion. Curr Biol 15: 1185-1195.

Vlachou D, Zimmermann T, Cantera R, Janse CJ, Waters AP, Kafatos FC 2004. Real-time in vivo analysis of malaria ookinete locomotion and mosquito midgut invasion. Cell Microbiol 6: 671-685.

Yajima M, Takada M, Takahashi N, Kikuchi H, Natori S, Oshima Y, Kurata S 2003. A newly established in vitro culture using transgenic Drosophila reveals functional coupling between the phospholipase A2-generated fatty acid cascade and lipopolysaccharide-dependent activation of the immune deficiency (IMD) pathway in insect immunity. Biochem J 371: 205-210. 